\title{
Effects of Loss Factor on Plane Wave Propagation through a Left-Handed Material Slab
}

\author{
C. SABAH* \\ Electrical and Electronics Engineering Department, University of Gaziantep \\ 27310, Gaziantep, Turkey
}

(Received February 26, 2008; revised version April 1, 2008)

\begin{abstract}
This article presents a theoretical and numerical discussion of the reflection and transmission analysis through a lossy left-handed material slab embedded between two semi-infinite dielectric media. The properties of the lossy left-handed material are given in detail and the required equations for the electromagnetic plane wave propagation are derived to solve the problem. The main important contribution of this article is the characterization of left-handed material that is different from its counterpart known in the literature. After introducing the left-handed material slab, the analytical solution is found for the powers carried by an electromagnetic wave. Then, the reflected, the transmitted, and the loss power are computed in terms of the incidence angle, the frequency, and the slab thickness in the numerical results with the emphasis on the loss factor.
\end{abstract}

PACS numbers: 04.30.Nk, 41.20.Jb, 78.20.Ci

\section{Introduction}

The left-handed materials (LHM) which have a negative permittivity and permeability have been receiving an important attention in the electromagnetics' community due to the possibility of construction at the microwave, millimeterwave, and optical frequencies. Such materials are not natural, but they are artificially manufactured at the given frequencies to illustrate the negative permeability and permittivity. First of all, the LHM were theoretically proposed by Veselago in 1968 [1]. In his study, this remarkable material was defined and the general properties of wave propagation in the LHM medium are presented. Then, Pendry et al. showed how the negative-permittivity materials could be realized from arrays of wires in 1996 [2] and negative-permeability materials from arrays of split

*e-mail: sabah@gantep.edu.tr 
rings in 1999 [3]. Recently, Smith et al. produced the composite materials having a negative permittivity and permeability using the combinations of split rings and wires, and did several microwave experiments to illustrate that the properties of this material are unlike from any existing material in 2000 [4]. Shelby et al. performed the experimental observation for the negative index of refraction on the LHM at microwave frequencies in 2001 [5]. Conceptual and speculative ideas for potential applications of the LHM were suggested, and physical remarks and intuitive comments were provided in [6]. Kong studied the electromagnetic wave interaction with stratified LHM isotropic media [7]. In his study, general formulations for the wave interaction with stratified media were given, and the field solutions of guided waves in stratified media were obtained. The reflected and transmitted powers due to the interaction of electromagnetic waves with a lossless LHM slab were presented by Sabah et al. in 2006 [8]. The transfer matrix method was used in the analysis to find the formulations in closed form. Also, the effects of the structure parameters, incidence angle and the frequency on the reflected and the transmitted powers were analyzed numerically.

In this study, the loss effects on the wave propagation through a lossy LHM slab are investigated in detail. Specifically, the reflected, the transmitted, and the loss power are computed and presented both analytically and numerically. The lossy LHM slab is sandwiched between the two semi-infinite dielectric media. The incident electric field is assumed as an electromagnetic plane wave with the perpendicular polarization. The electric and magnetic fields in all regions are determined using the Maxwell equations. Then, imposing the boundary conditions at the interfaces, the relationship among the incident, reflected and transmitted power can be found easily. Finally, some examples and case studies can be found in the numerical results to show the effects of the loss factor on the mentioned powers.

\section{Theory and analysis}

Let us consider an incident electric field of perpendicular polarization to be impinging on the LHM slab with the incidence angle $\theta_{\mathrm{i}}$ as shown in Fig. 1. In the analysis, $\exp (\mathrm{j} \omega t)$ time dependence is assumed and it is suppressed throughout this work. The total perpendicularly polarized electric fields in all regions can be written as

$$
\boldsymbol{E}=\left\{\begin{array}{l}
\boldsymbol{a}_{y} E_{\mathrm{i}}\left[\exp \left(-\gamma_{\mathrm{i} x} x\right)\right]\left[\exp \left(-\gamma_{\mathrm{i} z} z\right)+R \exp \left(\gamma_{\mathrm{i} z} z\right)\right], \quad z \leq 0, \\
\boldsymbol{a}_{y}\left[\exp \left(-\gamma_{\mathrm{s} x} x\right)\right]\left[A \exp \left(-\gamma_{\mathrm{s} z} z\right)+B \exp \left(\gamma_{\mathrm{s} z} z\right)\right], \quad 0 \leq z \leq d, \\
\boldsymbol{a}_{y} T E_{\mathrm{i}} \exp \left(-\left(\gamma_{\mathrm{t} x} x+\gamma_{\mathrm{i} z}(z-d)\right)\right), \quad z \geq d,
\end{array}\right.
$$

where $\gamma_{\mathrm{i}}, \gamma_{\mathrm{s}}, \gamma_{\mathrm{t}}$ are the propagation constants, $E_{\mathrm{i}}$ is the amplitude of the incident electric field, $A$ and $B$ are the amplitudes of the electric fields in the LHM slab, $\mathrm{d}$ is the slab thickness, $R$ and $T$ are the complex reflection and transmission coefficients. In all representations, the subscripts $\mathrm{i}, \mathrm{s}$, and $\mathrm{t}$ stand for the incident, 


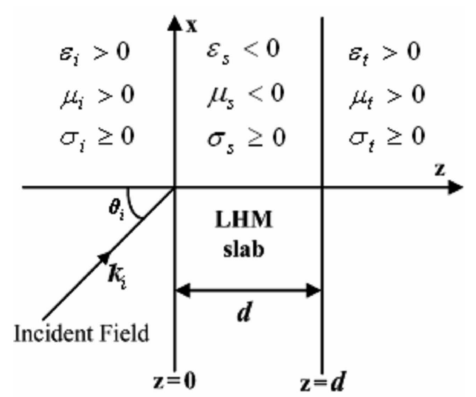

Fig. 1. The geometry of the lossy LHM slab between two lossy dielectric media.

slab, and transmitted media, respectively. In general, the propagation constant $\gamma$ in the lossy materials is expressed as

$$
\gamma=\mathrm{j} k_{\mathrm{c}}=\mathrm{j} \beta-\alpha=\mathrm{j} \omega \sqrt{\mu_{0} \mu_{\mathrm{r}} \varepsilon_{0}\left(\varepsilon_{\mathrm{r}}+\mathrm{j} \frac{\sigma}{\omega \varepsilon_{0}}\right)},
$$

where $k_{\mathrm{c}}$ is the complex wave number $\left(k_{\mathrm{c}}=\beta+\mathrm{j} \alpha\right), \alpha$ is the attenuation constant, $\beta$ is the phase constant, $\mu_{0}$ and $\varepsilon_{0}$ are the free-space permeability and permittivity, $\mu_{\mathrm{r}}$ and $\varepsilon_{\mathrm{r}}$ are the relative permeability and the relative permittivity of the lossy material, and $\sigma$ is the electric conductivity of the lossy material, respectively. From Eq. (2) we have

$$
\begin{aligned}
& \alpha^{2}-\beta^{2}=-\omega^{2} \mu_{0} \mu_{\mathrm{r}} \varepsilon_{0} \varepsilon_{\mathrm{r}}=-\omega^{2} \mu \varepsilon, \\
& 2 \alpha \beta=\omega \mu_{0} \mu_{\mathrm{r}} \sigma=\omega \mu \sigma,
\end{aligned}
$$

where $\mu$ and $\varepsilon$ are the permeability and permittivity of the given material. If we solve Eq. (3) and Eq. (4) together for $\alpha$ and $\beta$, four possible solutions are obtained as

$$
\begin{aligned}
& \alpha=\mp \omega \sqrt{\mu \varepsilon} \sqrt{\frac{1}{2}\left[\sqrt{1+\left(\frac{\sigma}{\omega \varepsilon}\right)^{2}}-1\right]}, \\
& \beta=\mp \omega \sqrt{\mu \varepsilon} \sqrt{\frac{1}{2}\left[\sqrt{1+\left(\frac{\sigma}{\omega \varepsilon}\right)^{2}}+1\right]} .
\end{aligned}
$$

In this study, the phase constant is positive in the right-handed material $(\mathrm{RHM})\left(\beta_{\mathrm{RHM}}>0\right)$ and it is negative in the LHM $\left(\beta_{\mathrm{LHM}}<0\right)$. However, the attenuation constant is always positive or zero in RHM and LHM $(\alpha \geq 0)$. Thus, the complex wave number can be written under the consideration of Eq. (2) as follows:

$$
\begin{aligned}
& k_{\mathrm{c}}=\beta+\mathrm{j} \alpha \quad(\text { for } \mathrm{RHM}), \\
& k_{\mathrm{c}}=-\beta+\mathrm{j} \alpha \quad(\text { for LHM), }
\end{aligned}
$$

where $\alpha$ and $\beta$ are the positive real numbers in Eq. (7) and Eq. (8). As mentioned 
before, the phase constant is negative in LHM. For example, in the lossless case where $\sigma=0$ and $\alpha=0$, the phase constant becomes $\beta=-\omega(\mu \varepsilon)^{1 / 2}$ which is the common knowledge assumed in many studies [1-8].

Now, imposing the boundary conditions at the interfaces the reflection and transmission coefficients mentioned in Eq. (1) can be obtained easily. Thus, they can be expressed as

$$
\begin{aligned}
R & =\frac{R_{01}+R_{12} \exp \left(\mathrm{j} 2 k_{\mathrm{cs} z} d\right)}{1+R_{01} R_{12} \exp \left(\mathrm{j} 2 k_{\mathrm{cs} z} d\right)}, \\
T & =\frac{4 \exp \left(\mathrm{j} k_{\mathrm{cs} z} d\right)}{\left(1+r_{01}\right)\left(1+r_{12}\right)\left[1+R_{01} R_{12} \exp \left(\mathrm{j} 2 k_{\mathrm{cs} z} d\right)\right]},
\end{aligned}
$$

in which $R_{01}$ and $R_{12}$ are the Fresnel reflection coefficients

$$
R_{01}=\frac{1-r_{01}}{1+r_{01}}, \quad R_{12}=\frac{1-r_{12}}{1+r_{12}},
$$

where for TE waves

$$
r_{01}=\frac{\mu_{\mathrm{i}} k_{\mathrm{cs} z}}{\mu_{\mathrm{s}} k_{\mathrm{ci} z}}, \quad r_{12}=\frac{\mu_{\mathrm{s}} k_{\mathrm{ct} z}}{\mu_{\mathrm{t}} k_{\mathrm{cs} z}}
$$

and for TM waves

$$
r_{01}=\frac{\varepsilon_{\mathrm{i}} k_{\mathrm{cs} z}}{\varepsilon_{\mathrm{s}} k_{\mathrm{ci} z}}, r_{12}=\frac{\varepsilon_{\mathrm{s}} k_{\mathrm{ct} z}}{\varepsilon_{\mathrm{t}} k_{\mathrm{cs} z}} .
$$

Here, $k_{\mathrm{c} m z}(m=\mathrm{i}, \mathrm{s}, \mathrm{t})$ is the $z$-component of the complex wave number and it can be written as for the configuration given in Fig. 1:

$$
k_{\mathrm{c} m z}=\sqrt{\omega^{2} \mu_{0} \mu_{\mathrm{r} m} \varepsilon_{0}\left(\varepsilon_{\mathrm{r} m}+\mathrm{j} \frac{\sigma_{m}}{\omega \varepsilon_{0}}\right)-k_{\mathrm{ci} x}^{2}},
$$

where $k_{\mathrm{ci} x}\left(k_{\mathrm{ci} x}=k_{\mathrm{ci}} \sin \theta_{\mathrm{i}}\right)$ is the $x$-component of the complex wave number of the incident electric field.

The $z$-component of the incident, the reflected, and the transmitted power can be represented as follows:

$$
P_{\mathrm{i} z}=\left|\frac{k_{\mathrm{ci} z}}{\mu_{\mathrm{i}}} E_{\mathrm{i}}^{2}\right|, \quad P_{\mathrm{r} z}=\left|\frac{k_{\mathrm{ci} z}}{\mu_{\mathrm{i}}} E_{\mathrm{r}}^{2}\right|, \quad \text { and } \quad P_{\mathrm{t} z}=\left|\frac{k_{\mathrm{ct} z}}{\mu_{\mathrm{t}}} E_{\mathrm{t}}^{2}\right|,
$$

where $E_{\mathrm{r}}\left(=R E_{\mathrm{i}}\right)$ is the amplitude of the reflected electric field and $E_{\mathrm{t}}\left(=T E_{\mathrm{i}}\right)$ is the amplitude of the transmitted electric field. If the incident electric field is normalized to unity, the conservation of the power allows defining the loss power as

$$
P_{\text {loss }}=1-\left(\left|R^{\mathrm{TE}, \mathrm{TM}}\right|^{2}+\left|\frac{k_{\mathrm{ct} z} \mu_{\mathrm{i}}}{k_{\mathrm{ci} z} \mu_{\mathrm{t}}}\right|\left|T^{\mathrm{TE}, \mathrm{TM}}\right|^{2}\right) .
$$

Let us note that LHM mentioned in this study is defined using conventional lossy dispersive medium parameters as seen in Eqs. (2), (5), and (6). In the literature, LHMs are described using hypothetical medium, Lorentz, Drude, cold plasma, and etc. parameters as in [9-15]. Consequently, LHM is identified using different approach distinct from the classifications presented in the literature. In conclusion, the suggested approach is the main significant contribution of this 
article and it is not found in the literature review of the electromagnetic plane wave propagation in the presence of LHM studies.

\section{Numerical results}

In this section, the effects of the loss factor, $\sigma$, on the behavior of the powers are presented numerically for the perpendicular polarization (TE wave). To verify the results used in these computations, firstly the conservation of power given in Eq. (16) is satisfied for all examples. Secondly, a transmission line equivalent model is derived for the structure given in Fig. 1 [16]. It is seen that both methods give the same numerical values for all computations. Thus, the results are verified by means of two concepts, the conservation of power and the transmission line equivalent model.

The reflected $\left(P_{\mathrm{r}}\right)$, the transmitted $\left(P_{\mathrm{t}}\right)$, and the loss $\left(P_{\text {loss }}\right)$ power are calculated as a function of the incidence angle, the frequency and the slab thickness. In the calculations, the operation frequency was chosen by an arbitrary decision to be $f_{0}=10.8 \mathrm{GHz}$ to explore the characteristics of the mentioned powers in the microwave frequency band. The first and the last materials given in Fig. 1 are assumed to be lossless materials $\left(\sigma_{\mathrm{i}}=\sigma_{\mathrm{t}}=0\right)$ and the permeabilities of them are equal to the permeability of the free space, $\mu_{\mathrm{i}}=\mu_{\mathrm{t}}=\mu_{0}$.

In the first example, it is considered that the first and the last materials are glass and mica whose relative permittivities are 8.0 and 7.0, respectively. The permeability and permittivity of the LHM slab are selected to be $\mu_{\mathrm{s}}=-1.5541 \mu_{0}$ and $\varepsilon_{\mathrm{s}}=-4.4739 \varepsilon_{0}$, respectively, to provide the approximate impedance matching between the LHM slab and the last material for the given arrangement. The slab thickness is assumed to be a quarter-wavelength long at the operation frequency $f_{0}$. Figure 2 presents the reflected, the transmitted, and the loss power for the perpendicular polarization as a function of the incidence angle for TE wave. From Fig. $2 \mathrm{a}$, the reflected power changes from zero to 0.4 for the incidence angle less than $68.79^{\circ}$ for all electric conductivity values. This is the critical angle for the given configuration. When the incidence angle greater than or equal to this angle, $P_{\mathrm{r}}$ becomes unity, $P_{\mathrm{t}}$ and $P_{\text {loss }}$ become zero. From Fig. $2 \mathrm{~b}, P_{\mathrm{t}}$ almost reaches the value of unity at the incidence angle of $37^{\circ}$, when the electric conductivity is $\sigma_{\mathrm{s}}=10^{-4} \mathrm{~S} / \mathrm{m}$. Therefore, this angle is the quasi-Brewster angle in which the reflection and the loss power are nearly zero and the transmitted power is about the unity for this structure. From Fig. 2c, as the conductivity increases, $P_{\text {loss }}$ also increases, but $P_{\mathrm{t}}$ decreases as seen in Fig. 2b. Furthermore, $P_{\text {loss }}$ closes to zero when $\sigma_{\mathrm{s}}=10^{-4} \mathrm{~S} / \mathrm{m}$. As it is seen, $P_{\mathrm{r}}$ is not much affected from the changing in the conductivity for this configuration. However, $P_{\mathrm{t}}$ and $P_{\text {loss }}$ are so much affected from the variation in the conductivity.

As the second example, frequency response of the powers is considered. The relative permittivities of the first and the last materials are selected to be 1.0 (free-space) and 7.0 (mica), respectively. The permittivity and permeability of the 

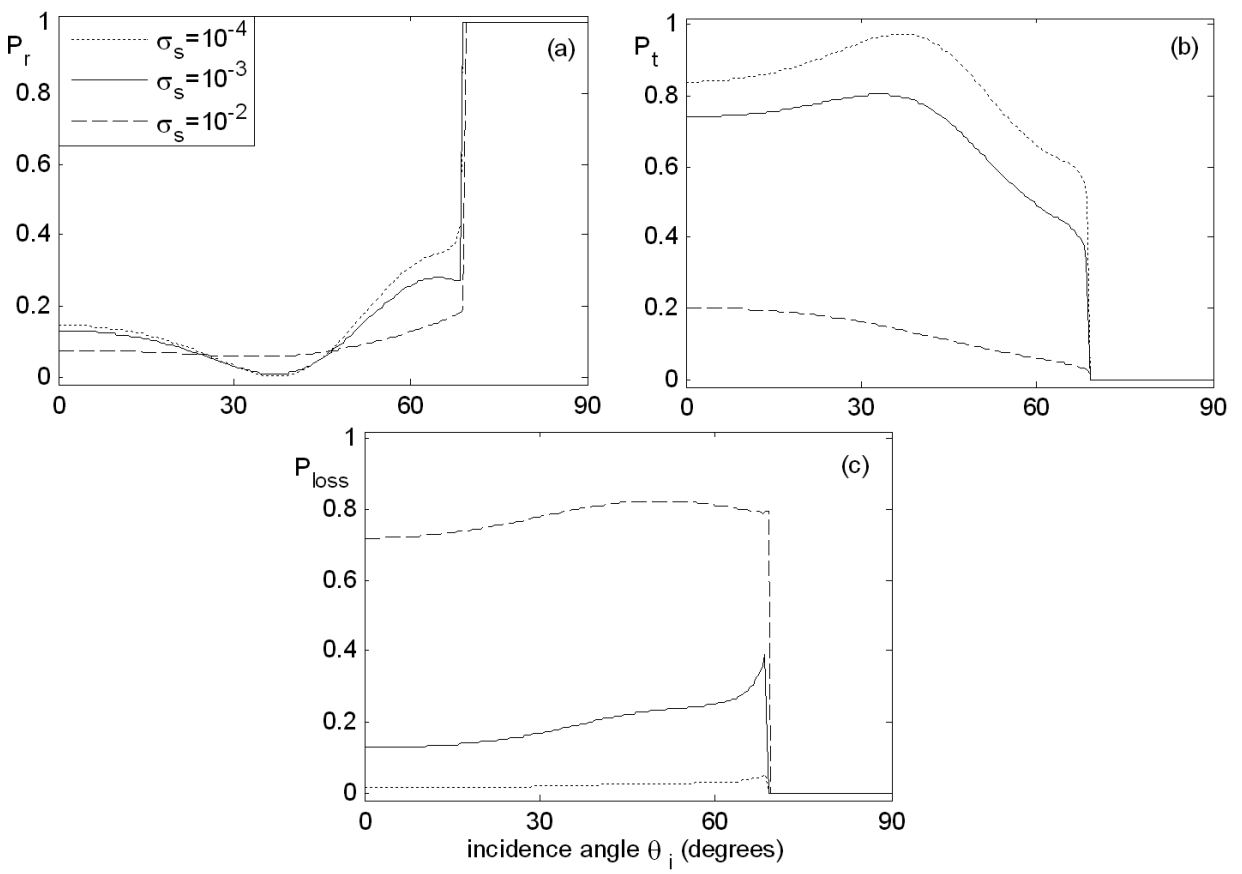

Fig. 2. The reflected (a), the transmitted (b), and the loss power (c) for TE wave as a function of the incidence angle.

LHM slab, and the slab thickness are the same with the previous example. The angle of incidence is assumed $30^{\circ}$. The reflected, the transmitted, and the loss power for TE wave versus the frequency are depicted in Fig. 3. As it can be seen from Fig. 3a, $P_{\mathrm{r}}$ changes periodically in the different range when $\sigma_{\mathrm{s}}=10^{-3} \mathrm{~S} / \mathrm{m}$ and $\sigma_{\mathrm{s}}=10^{-4} \mathrm{~S} / \mathrm{m}$. On the other hand, when $\sigma_{\mathrm{s}}=10^{-2} \mathrm{~S} / \mathrm{m}$, it shows a decreasing inclination up to $5 \mathrm{GHz}$ and after that it changes again periodically. The frequency response of $P_{\mathrm{t}}$ is remarkably sinusoidal for the small values of the electric conductivity (i.e. $\sigma_{\mathrm{s}}=10^{-3} \mathrm{~S} / \mathrm{m}$ and $\sigma_{\mathrm{s}}=10^{-4} \mathrm{~S} / \mathrm{m}$ ). But, it is about the value of 0.2 when $\sigma_{\mathrm{s}}=10^{-2} \mathrm{~S} / \mathrm{m}$. From Fig. 3c, $P_{\text {loss }}$ increases up to $5 \mathrm{GHz}$ and after this frequency it shows a slightly periodic behavior for the high conductivity value given in this computation. It varies as a slightly periodic function when $\sigma_{\mathrm{s}}=10^{-3} \mathrm{~S} / \mathrm{m}$ and also it is about zero when $\sigma_{\mathrm{s}}=10^{-4} \mathrm{~S} / \mathrm{m}$. Thus, the loss factor $\sigma$ which changes the behavior of the powers plays an important role in the variation of them. It is obviously seen from the figure that the high percentage of the incident power appears as a loss in the LHM slab when the conductivity is high (i.e. $\sigma_{\mathrm{s}}=10^{-2} \mathrm{~S} / \mathrm{m}$ ). At this condition, $P_{\mathrm{r}}$ is less affected from the changes in the conductivity on the contrary of $P_{\mathrm{t}}$ and $P_{\text {loss }}$.

In the last example, the effect of the slab thickness on the powers is observed. The permittivity and permeability of the all materials are the same with the second example. The incidence angle is $60^{\circ}$. Figure 4 points out the variation of the slab 


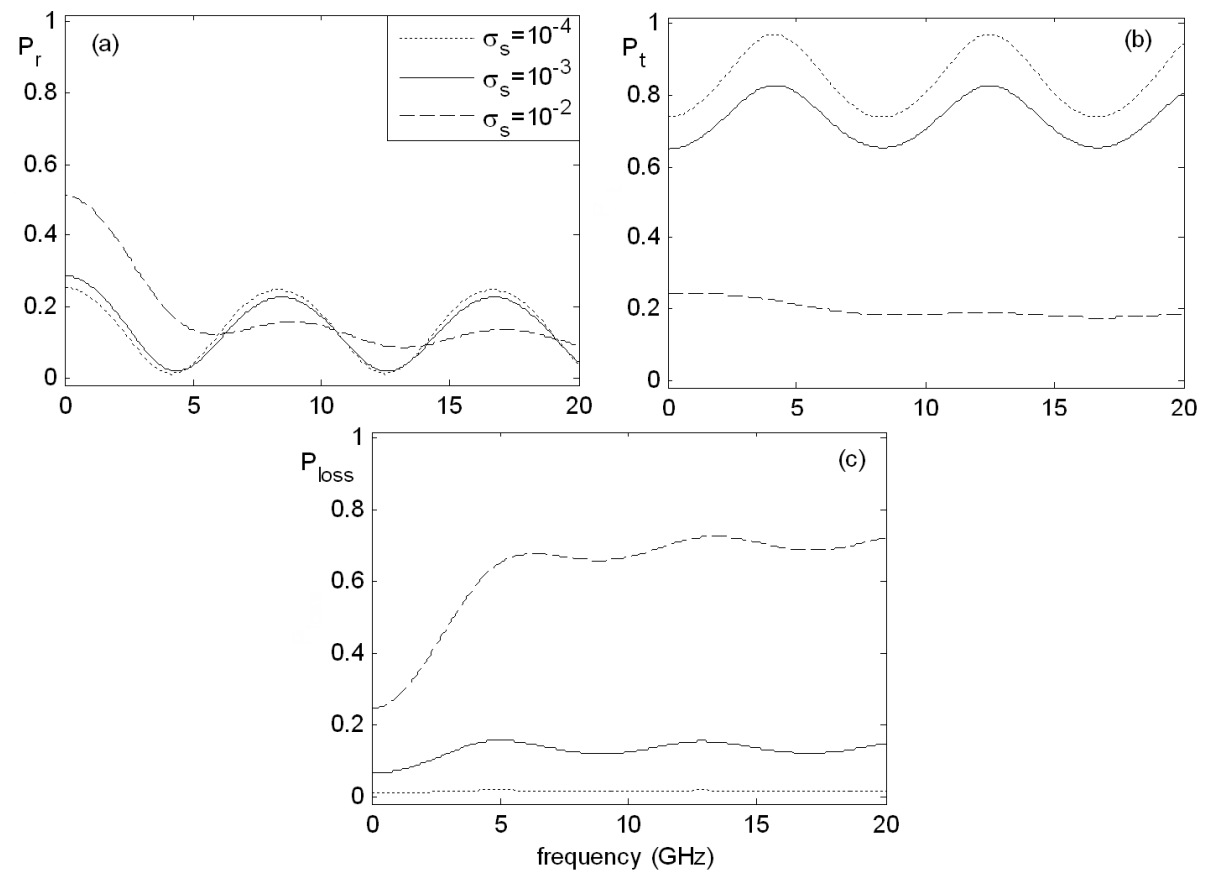

Fig. 3. The reflected (a), the transmitted (b), and the loss power (c) for TE wave versus frequency.

thickness in $P_{\mathrm{r}}, P_{\mathrm{t}}$, and $P_{\text {loss }}$ for TE wave. Figure 4 a corresponds to the reflected power. It is observed that the characteristic of $P_{\mathrm{r}}$ for this arrangement behaves like an oscillatory function for all electric conductivity values. Let us note that $P_{\mathrm{r}}$ does not change significantly after $d=1.5 \mathrm{~cm}$ and it is around 0.3 , when $\sigma_{\mathrm{s}}=$ $10^{-2} \mathrm{~S} / \mathrm{m}$. In addition, $P_{\mathrm{t}}$ has an almost periodically decline characteristic when $\sigma_{\mathrm{s}}=10^{-4} \mathrm{~S} / \mathrm{m}$. It shows an oscillatory decreasing behavior when $\sigma_{\mathrm{s}}=10^{-3} \mathrm{~S} / \mathrm{m}$. On the other hand, at the same value of the conductivity, $P_{\text {loss }}$ shows an oscillatory increasing behavior. However, $P_{\mathrm{t}}$ decays like an exponential function and $P_{\text {loss }}$ varies like an increasing function for the high value of the conductivity given in this example. Furthermore, the loss power changes around zero for the smallest value of the conductivity. It is observed that the reflected and the transmitted power are directly related with the loss factor. Finally, the same explanation as in the previous example can be stated when the conductivity is high.

It is confirmed that the similar numerical results can be obtained as shown in Figs. 2-4 for the incident wave with the parallel polarization. 

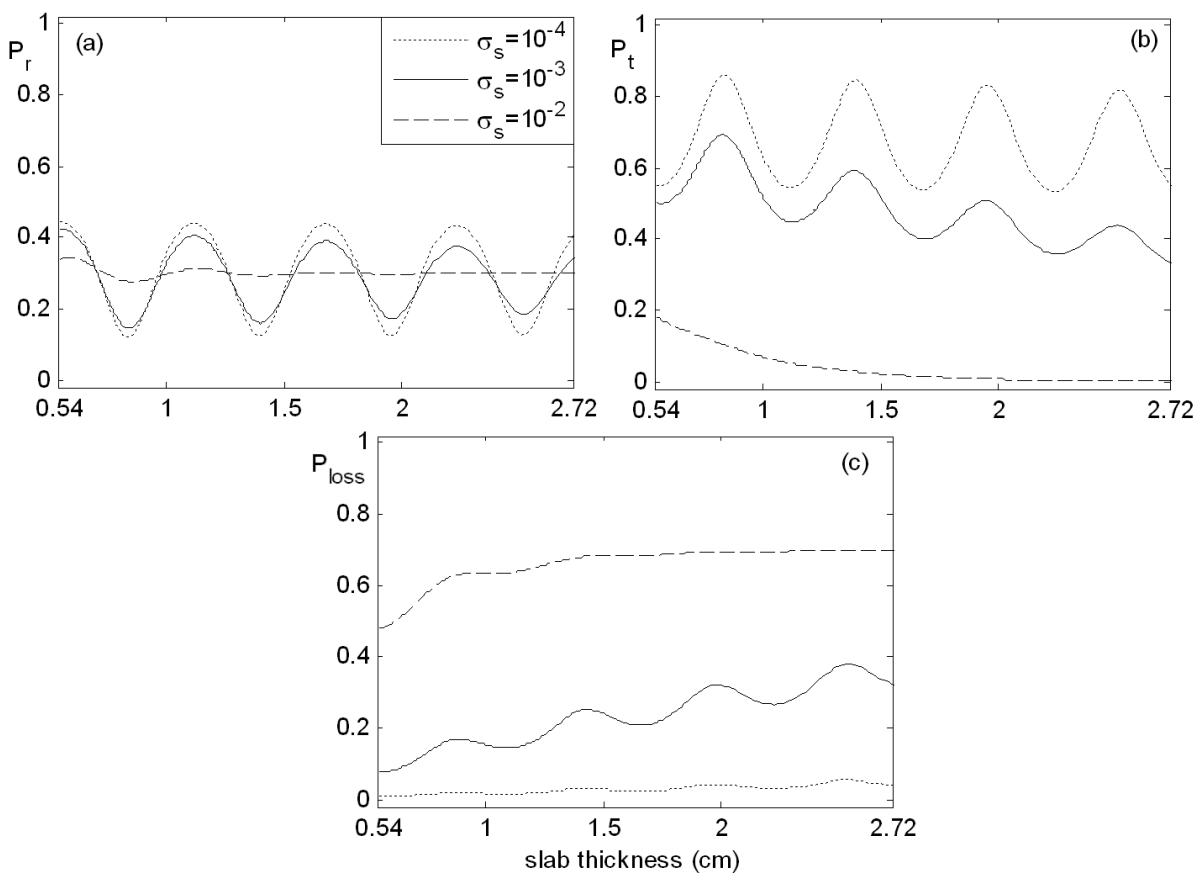

Fig. 4. The reflected (a), the transmitted (b), and the loss power (c) for TE wave against the slab thickness.

\section{Conclusions and discussions}

In this work, the reflection and transmission characteristics of the electromagnetic radiation propagation through a lossy LHM slab are studied in detail with the effects of the loss factor. First of all, the structure of the lossy LHM slab between two lossy dielectric media is arranged and the structure parameters are defined. Then, the required equations for the propagation constant, the complex wave number, the phase constant, and the attenuation constant are derived. Later, the reflection and transmission coefficients are obtained in the closed form. After that, the incident, the reflected, and the transmitted power are given and the conservation of the power is determined in the existence of the loss power. Finally, the reflected, the transmitted and the loss power as a function of the incidence angle, the frequency, and the slab thickness are studied numerically to observe the effects of the loss factor on them. As it can be seen from the theoretical and the numerical results, if the loss factor changes, the characteristic of the powers will be affected from this change. The amount of this change depends on the characteristic of the structure. Thus, we can say that the loss factor which changes the behavior of the powers plays an important role in the variation of these powers. The loss effects (low and high power losses) can easily be observed from the numerical results and they can be optimized in the optimization process by re-arranging 
the structure parameters. Similar behaviors were observed in Ref. [16]. In that study, LHM was defined using the Lorentz and Drude parameters. Therefore, it can be said that the results achieved in this study agree and are compatible with the results obtained in Ref. [16]. Therefore, the suggested approach can be used to define and characterize LHMs. In addition to these explanations, the Fabry-Perot resonances can be seen in the obtained numerical results. The reflections can be nearly ignored and it can be achieved to the total transmission at this resonance condition. Furthermore, to obtain high transmission, some tunable structures can be designed by tuning the fraction of the thickness of LHM slab. Thus, the results obtained here can be helpful to design new devices, apparatuses and/or components at the millimeter wave, optical, and microwave regimes. Furthermore, these results open a way to think how the availability of the losses in LHM materials will change the functionality of a device with LHM slabs. Moreover, this study will make a foundation for future works and provide some insight into the potential applications of LH materials and/or LHM slab.

\section{References}

[1] V.G. Veselago, Sov. Phys.-Usp. 10, 509 (1968).

[2] J.B. Pendry, A.J. Holden, W.J. Stewart, I. Youngs, Phys. Rev. Lett. 76, 4773 (1996).

[3] J.B. Pendry, A.J. Holden, D.J. Robbins, W.J. Stewart, IEEE Trans. Microwave Theory Techn. 47, 2075 (1999).

[4] D.R. Smith, W.J. Padilla, D.C. Vier, S.C. Nemat-Nasser, S. Schultz, Phys. Rev. Lett. 84, 4184 (2000).

[5] R.A. Shelby, D.R. Smith, S. Schultz, Science 292, 77 (2001).

[6] N. Engheta, in: Advances in Electromagnetics of Complex Media and Metamaterials, NATO Science Series, Proc. NATO Advanced Research Workshop in Marrakech (Bianisotropics'2002), Eds. S. Zouhdi, A.H. Sihvola, M. Arsalane, Kluwer Academic Publishers, Inc., 2002.

[7] J.A. Kong, Prog. Electromagn. Res. 35, 1 (2002).

[8] C. Sabah, G. Ögücü, S. Uckun, J. Optoelectron. Adv. Mater. 8, 1925 (2006).

[9] J.B. Pendry, Phys. Rev. Lett. 85, 3966 (2000).

[10] T.J. Cui, J.A. Kong, Phys. Rev. B 70, 205106 (2004).

[11] D.R. Smith, N. Kroll, Phys. Rev. Lett. 85, 2933 (2000).

[12] D.R. Smith, D.C. Vier, N. Kroll, S. Schultz, Appl. Phys. Lett. 77, 2246 (2000).

[13] R.W. Ziolkowski, E. Heyman, Phys. Rev. E 64, 056625 (2001).

[14] C. Sabah, Opt. Appl. 37, 123 (2007).

[15] C. Sabah, S. Uckun, Opto-Electron. Rev. 15, 133 (2007).

[16] D.H. Staelin, A.W. Morgenthaler, J.A. Kong, Electromagnetic Waves, Prentice Hall International Editions, 1994. 